alkali metals follow a curious order, namely, lithium, rubidium, potassium, sodium. It is interesting, however, to observe that this is the order of the heats of formation of the several ions in aqueous solution. In order to illustrate this fact the heat of formation of the chlorides of the four metals in aqueous solution are given in the last column.

\begin{tabular}{|c|c|c|c|}
\hline$P$ & $\begin{array}{l}\text { TABLE IV. } \\
\text { Potential against } \\
\text { N. } \mathrm{E} \text {. }\end{array}$ & $\begin{array}{l}\text { Potential against } \\
\text { hydrogen. }\end{array}$ & $\begin{array}{l}\text { Heat of formation } \\
\text { of chlorides. }\end{array}$ \\
\hline $\mathrm{Li}$. . & . 3.305 & 3.027 & 102000 \\
\hline $\mathrm{Rb}$. & 3.205 & 2.928 & 101000 \\
\hline $\mathrm{K}$. & 3.203 & 2.925 & 101000 \\
\hline$a \ldots \ldots \ldots \ldots \ldots \ldots \ldots$ & 2.993 & 2.715 & 96000 \\
\hline
\end{tabular}

The heat of formation of aqueous cesium chloride is given as 105,000 calories, and if this figure is correct we might predict the potential of cesium to be higher than that of any of the other alkali metals.

We wish to express our indebtedness to the Bache Fund of the National Academy for financial aid in this series of investigations.

Berkeley CaL.

[Contribution from Chemical Laboratory, Bryn Mawr College.]

\title{
A STUDY OF ALCOHOLIC SOLUTIONS OF CADMIUM IODIDE.
}

By Frederick H. Getman and Vernette L. Gibbons.

Received June 11, 1915

\section{Conductance.}

The data for the conductance of cadmium iodide in alcoholic solutions was found to be very meager. Zelinsky and Krapiwin, ${ }^{1}$ and Jones and Carroll, ${ }^{2}$ measured the conductance of various solutions of cadmium iodide with methyl alcohol as the solvent, but there is great disagreement between the two sets of values, and when plotted neither set gives a smooth curve. Jones and Carroll ${ }^{2}$ also measured the conductance of cadmium iodide with ethyl alcohol as the solvent. As far as recorded, only one series of measurements was made in each case. It therefore seemed advisable to redetermine the conductance of cadmium iodide in methyl and ethyl alcoholic solutions.

In the work, the results of which are here given, extreme care was taken to avoid, as far as possible, all known sources of error. The purest cadmium iodide obtainable was powdered and allowed to stand in a desiccator over phosphorous pentoxide for several days. The alcohols were dehydrated over pure lime for several weeks and then distilled, using a fractionating column and collecting in a dry receiver protected from the gases and moisture of the atmosphere by means of a soda-lime tube. The boiling point of the ethyl alcohol was $77.3^{\circ}$ at $743.1 \mathrm{~mm}$. and the boiling point

I Z. physik. Chem., 21, 35-52 (1896).

${ }^{2}$ Bull. Carnegie Inst., 80, $4^{1-73}$ (1907). 
of the methyl alcohol was $64.2^{\circ}$ to $64.5^{\circ}$ at $75^{2} \mathrm{~mm}$. The measurements of the electrical conductance were made by means of the well-known Kohlrausch apparatus, a Leeds and Northrup cylindrical bridge being used. The cell was of the ordinary Arrhenius form, closed with a tightfitting ebonite cover. Its constant was found to be 16.27 and did not alter during the progress of the conductance measurements.

The solutions were made up either by direct weighing or by dilution from a stronger solution. The flasks and burets used were carefully calibrated. In several cases duplicate measurements were made with solutions made up by direct weighing and by dilution from a more concentrated

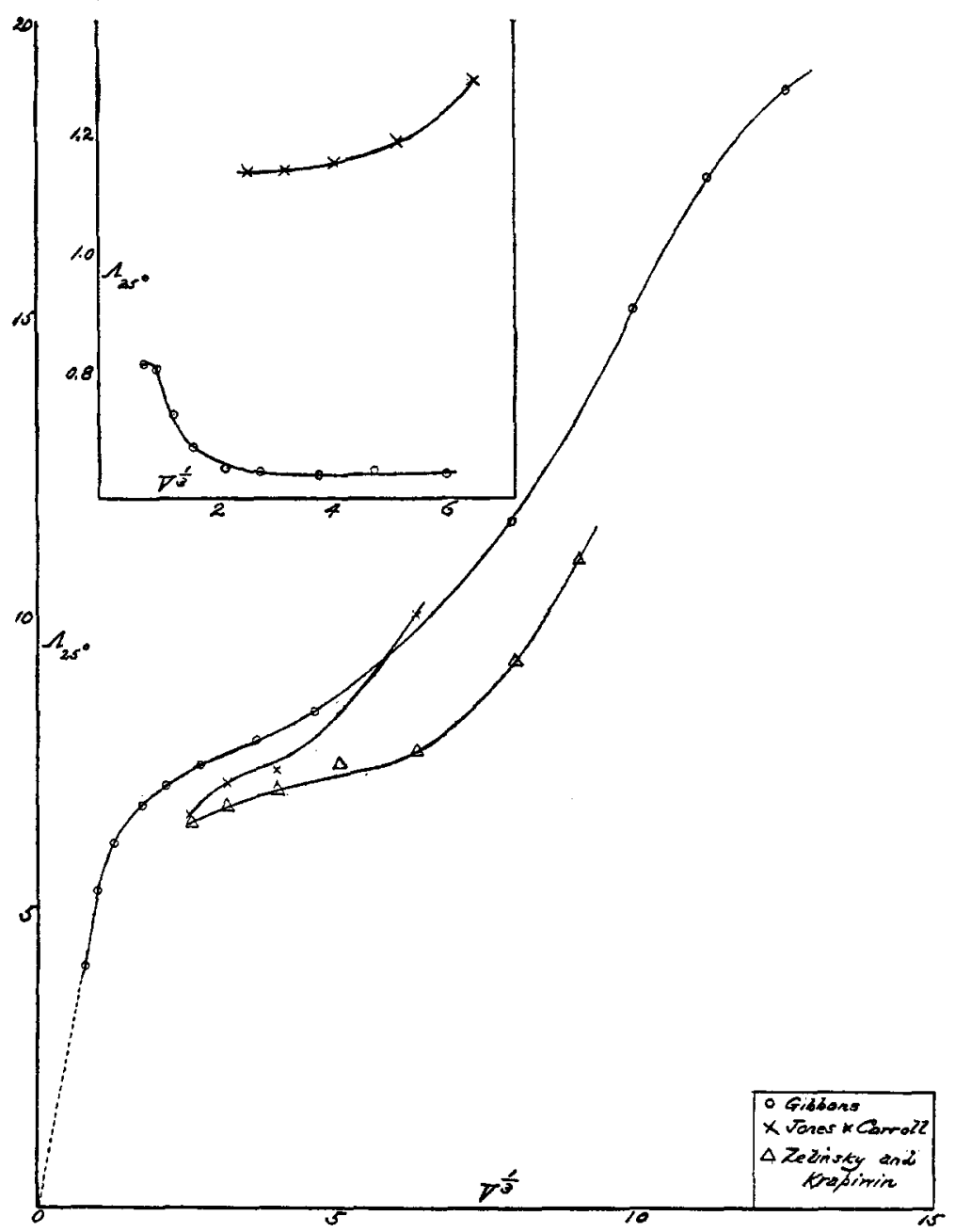

Fig. I. 
solution, and in no case was a solution diluted more than twice. The cell was always filled twice and duplicate readings taken, so that at least four measurements were made with each solution. These results corroborate each other and the curves are quite smooth, as is shown by Fig. I, plotted from the data given in Table I.

\section{TABLE I.}

SOLUTIONS IN METHYL, ALCOHOL.

$\begin{array}{lcclcl}m . & \mathrm{V} . & \Delta_{25^{\circ}} & m . & \mathrm{V} . & \boldsymbol{A}_{25}{ }^{\circ} \\ 2.0 & 0.5 & 4.14 & 2.0 & 0.5 & 0.825 \\ 1.0 & \mathrm{I} & 5.40 & 1.0 & 1 & 0.817 \\ 0.5 & 2 & 6.20 & 0.5 & 2 & 0.740 \\ 0.2 & 5 & 6.82 & 0.25 & 4 & 0.684 \\ 0.1 & 10 & 7.14 & 0.1 & 10 & 0.650 \\ 0.05 & 20 & 7.49 & 0.05 & 20 & 0.644 \\ 0.02 & 50 & 7.89 & 0.02 & 50 & 0.639 \\ 0.01 & 100 & 8.39 & 0.01 & 100 & 0.646 \\ 0.005 & 200 & 9.33 & 0.005 & 200 & 0.640 \\ 0.002 & 500 & 11.58 & & & \\ 0.001 & 1000 & 15.14 & & & \\ 0.0007 & 1428.6 & 17.35 & & & \\ 0.0005 & 2000 & 18.82 & & & \end{array}$

The conductance curve for methyl alcoholic solutions of cadmium iodide is complicated, indicating complex conditions in the solutions; the curve for the ethyl alcoholic solutions is the exact reverse of what one would naturally expect. It has been shown by Jones and Carroll ${ }^{1}$ that, when water is mixed with the alcohol as a solvent, the conductance of the solute is increased. It is obvious therefore, that the lower values obtained by us for the ethyl alcoholic solutions cannot be caused by the absorption of moisture by the solutions. The values obtained by us with solutions in methyl alcohol are, it is true, higher than those obtained by the other observers, but the fact that several points on the curve were corroborated by measurements of solutions made up by direct weighing and by dilution confirms the accuracy of the work. In the case of the $0.05 \mathrm{~N}$ concentration three solutions were used; one made up from a $N$ solution and two by direct weighing, using alcohol obtained by two different distillations.

Jones and Getman ${ }^{2}$ measured the molecular rise in the boiling point of ethyl alcoholic solutions of cadmium iodide. If molecular association occurs, as was plainly indicated by their results, the degree of association would probably be greater at $25^{\circ}$, the temperature at which the conductance measurements were made, than at the boiling point of the solvent. If one studies the plotted values for methyl alcoholic solutions of cadmium iodide given by Zelinsky and Krapiwin and Jones and Carroll (Fig. I), one sees that although the values lie lower than those given by us, there is,

${ }^{1}$ Bull. Carnegie Inst., 80, 4I-73 (1907).

2 Am. Chem. J., 3I, 303-359 (I904). 
in general, a similar trend or complexity, which partially explains the lack of smoothness observed. By comparing the curves here given with the conductance curves for silver nitrate in nonaqueous solutions given by Gibbons and Getman, ${ }^{1}$ one sees certain similarities. The curve for the conductance of cadmium iodide, when ethyl alcohol is the solvent, is quite analogous to the middle portion of the curve for the conductance of silver nitrate, when aniline was the solvent, and an upward trend is noted in the curves of conductance of silver nitrate in acetone, in pyridine and in aniline, just as is noted in the more dilute concentrations of cadmium iodide when methyl alcohol is the solvent. Just as the anomalies in the conductance curves of silver nitrate have been explained on the basis of complexes existing in the solution, so may also be explained the anomalies here observed with alcoholic solutions of cadmium iodide.

That molecular complexes do exist in both aqueous and nonaqueous solutions of cadmium iodide is shown by the determination of the transport numbers, the molecular depression of the freezing point, and the molecular elevation of the boiling point. Hittorf ${ }^{2}$ measured the transport numbers for cadmium iodide in ethyl and amyl alcoholic solutions and obtained negative values for the cadmium ion. The values obtained with amyl alcoholic solutions were greater than those obtained with ethyl alcoholic solutions. $\mathrm{Jahn}^{3}$ and his students worked with cadmium iodide in aqueous solutions and found the transport number for the cation practically constant for concentrations between $0.005 N$ and $0.2 N$, while for higher concentrations the value diminished and at a concentration of $0.5 \mathrm{~N}$ the transport number for the cation was negative, $i$. e., -0.0003 . Hittorf, ${ }^{2}$ and Noyes and $\mathrm{Falk}^{4}$ considered these negative values for the transport number of the cation as proof of the existence of complex ions in the solutions. McBain, ${ }^{5}$ after taking into account all the data of measurements of conductance, freezing point, and transport numbers, concluded that in a o.I $N$ aqueous solution about $80 \%$ of the molecules were undissociated $\mathrm{CdI}_{2}$ and that about half the anion was $\mathrm{CdI}_{3}{ }^{\prime}$. From the values of the transport numbers, it is clear that for any given concentration the ethyl alcoholic solution will contain a larger proportion of complexes, both molecules and ions, than the corresponding aqueous solution, and by analogy it is safe to assume that in a methyl alcoholic solution the proportion of complexes will be between the values for aqueous and for ethyl alcoholic solutions.

In solutions of cadmium iodide in the al ohols several reactions may take place. The complexes $\mathrm{C}_{m}$ may decompose into simpler molecules, or they

1 This Journal, 36, 1630-1655 (1914).

2 Pogg. Ann., 106, 551, 554, 558 (1859).

${ }^{3} Z$. physik. Chem., 37, 673-712 (1901).

4 This Journal, 33, I460 (I9II).

5 . Electrochem., II, 222 (1905). 
may ionize to form complex ions $\mathrm{A}^{\prime}$ and $\mathrm{K}^{\prime}$, or the simple molecules may ionize to form simple ions $A$ and $K$. The form which the conductance curve takes depends entirely upon which of the three reactions predominates. In the more concentrated regions the complexes predominate and in the more dilute regions solvation plays its part. In the case of silver nitrate solutions, the complexes were known to be a combination of solute and solvent, and in the cases where abnormal conductance occurred the solvent had a very low dielectric constant and high viscosity. In the case of cadmium iodide solutions, the complexes are combinations of the molecules of the solute. The conductance of the ethyl alcoholic solutions is extremely weak, which indicates a very slight amount of ionic dissociation. The abnormalities observed in the ethyl alcoholic solution occur in the more concentrated regions, while those observed in the methyl alcoholic solutions occur in the more dilute regions. It would seem, therefore, that in the more concentrated ethyl alcoholic solutions the strong associative power of the solute offsets the effect of the moderately high dielectric constant of the solvent. In the more dilute methyl alcoholic solutions, it is possible that the dissociation of the simple molecules into ions predominates and causes the rapid upward slant of the curve. It is also possible that in these dilute solutions solvation plays its part. It is therefore quite apparent that the diminishing conductance with increasing dilution depends upon the complexity of the conducting complexes rather than upon the nature of their components.

\section{Density and Specific Refraction.}

In I9II-I9I2 Miss Gilroy, ${ }^{1}$ working in this laboratory, made a study of the relation between the density and the refractive index of aqueous solutions of cadmium iodide and calculated the specific refraction according to the Lorentz-Lorenz formula.

$$
r=\frac{\mathrm{I}}{p} \frac{n_{1}^{2}-\mathrm{I}}{n_{1}^{2}+\mathrm{I}} \cdot \frac{\mathrm{IOO}}{d_{1}}-\frac{n_{2}^{2}-\mathrm{I}}{n_{2}^{2}+\mathrm{I}} \cdot \frac{\mathrm{IOO}-p}{d_{2}} .
$$

( $r$ denotes the specific refraction of the solute at the temperature of the experiment, $p$ the per cent. of solute in the solution, $n_{1}$ and $n_{2}$ denote the refractive indices and $d_{1}$ and $d_{2}$ denote the densities of the solution and the solvent, respectively, at $20.5^{\circ}$.) She found the specific refraction at $25^{\circ}$ practically constant for the solutions employed (about $0.04 N$ to I.5 N).

A similar study of methyl and ethyl alcoholic solutions of cadmium iodide at $20.5^{\circ}$ was undertaken. A Sprengel-Ostwald pycnometer of about to cc. capacity was used and duplicate measurements were made with each solution. The densities were referred to water at $4^{\circ}$. The refractive indices were measured with the improved form of Pulfrich re-

1 Getman and Gilroy, Am. Chem. J., 48, I38-145 (1912). 
fractometer manufactured by Zeiss. The temperature was kept constant at $20.5^{\circ}=0.1^{\circ}$ by means of a Van Aubel thermostat. The light source was a sodium flame. As in the work with aqueous solutions, the LorentzLorenz formula was used for the calculation of the specific refraction, as it was found to give more uniform results.

TABLE II.

\begin{tabular}{|c|c|c|c|c|}
\hline & Cadmi & $\begin{array}{l}\text { TABLE I } \\
\text { lide in } I\end{array}$ & Icohol. & \\
\hline$m$. & $\%$ & $d$ & n. & r. \\
\hline o. & o. & 0.7942 & I. 32875 & \\
\hline 0.5 & I I . 555 & 0.8766 & I. 34300 & 0.1260 \\
\hline 0.2 & 4.602 & 0.8269 & I. $3345^{2}$ & 0.1212 \\
\hline O.I & 2.301 & 0.8099 & I. 33159 & O. $125^{8}$ \\
\hline 0.05 & I. 150 & $0.802 \mathrm{I}$ & I.33016 & 0.1223 \\
\hline 0.02 & 0.460 & 0.7969 & I. 32932 & o. I 537 \\
\hline 0.005 & O.II 5 & 0.7943 & I. 32887 & 0.2920 \\
\hline .0005 & 0.012 & 0.7942 & I. 32887 & 0.7270 \\
\hline
\end{tabular}

Cadmium Iodide in Ethyl Alcohol.

$\begin{array}{lcccc}0 . & 0 . & 0.789 \mathrm{I} & \mathrm{I} .36104 & \\ 2 . & 46.4 \mathrm{I} 2 & \mathrm{I} .1068 & \mathrm{I} .40964 & 0.1582 \\ \mathrm{I} . & 23.206 & 0.9484 & \mathrm{I} .385 \mathrm{I} 7 & 0.1373 \\ 0.5 & \mathrm{I} 1.6 \mathrm{I} 3 & 0.8688 & \mathrm{I} .37289 & 0.123 \mathrm{I} \\ 0.25 & 5.802 & 0.8292 & \mathrm{I} .36695 & 0.1142 \\ 0.2 & 4.64 \mathrm{I} & 0.82 \mathrm{IO} & \mathrm{I} .36559 & 0.1113 \\ 0 . \mathrm{I} & 2.32 \mathrm{I} & 0.8052 & \mathrm{I} .36355 & 0.1068 \\ 0.05 & 1.160 & 0.7973 & \mathrm{I} .36219 & 0.1004 \\ 0.02 & 0.464 & 0.7924 & \mathrm{I} .36148 & 0.0957 \\ 0.01 & 0.232 & 0.79 \mathrm{II} & \mathrm{I} .36136 & 0.0722 \\ 0.005 & 0.116 & 0.7900 & \mathrm{I} .36117 & 0.0833\end{array}$

Plotting the densities and refractive indices of the solutions against the concentrations shows the relationship in each case to be rectilinear. The specific refraction was calculated on the assumption that the volume of the solvent was in each case the same as the volume of the solution. The specific refraction was found to be practically constant for the more concentrated solutions in methyl alcohol, but for the less concentrated solutions the value increased rapidly with dilution. However, in the calculation of the specific refraction of a very dilute solution the sources of error may be very great. The specific refraction of the ethyl alcoholic solutions showed no constancy of value whatever, and instead of increasing with the dilution of the solution it rapidly diminished.

There was a possibility that the error introduced by assuming the volume of the solvent to be the same as the volume of the solution, might explain the difference. If in the one case there was expansion and in the other contraction, when the salt was dissolved, the value of the specific refraction would naturally be effected correspondingly. A determination of the volume changes on dissolving cadmium iodide in the two alcohols 
was carried out in the following way: A Ioo cc. calibrated measuring flask was weighed, filled to the mark with the pure alcohol at the temperature $20.5^{\circ}$. The weight of the alcohol was determined and the weight of cadmium iodide required to make $100 \mathrm{cc}$. of a given normality of solution was added to the alcohol in the flask. The expansion or the contraction was noted and its exact amount calculated from determinations of the density of each solution. The pycnometer, whose volume was about $20 \mathrm{cc}$., was filled three times with the given solution, thus three separate density determinations were made for each solution. Weights of cadmium iodide required to make Ioo cc. of $0.5 N$ and $0.2 N$ solution were taken for each alcohol. In every case there was expansion as is shown in Table III.

TABLE III.

\begin{tabular}{|c|c|c|c|c|c|}
\hline Solvent. & Conc. & $\begin{array}{l}\text { xpansion per } \\
100 \text { cc. of } \\
\text { solvent. }\end{array}$ & Solvent. & Conc. & $\begin{array}{l}\text { Expansion per } \\
\text { 100 cc. of } \\
\text { solvent. }\end{array}$ \\
\hline Ethyl alcohol.... & $\begin{array}{l}0.5 N \\
0.2 N\end{array}$ & $\begin{array}{l}1.38 \mathrm{cc} \\
0.46 \mathrm{cc}\end{array}$ & Methyl alcohol. & $\begin{array}{l}0.5 N \\
0.2 N\end{array}$ & $\begin{array}{l}\text { I. Io cc. } \\
0.33 \mathrm{cc} \text {. }\end{array}$ \\
\hline
\end{tabular}

Thus it is seen that any error introduced into the calculation because of volume changes would alter all the results in the same direction and hence cannot in any way explain the great differences observed in the values for the specific refraction of these solutions.

The fact that the variations in the specific refraction of these two classes of solutions run parallel to the variations in the conductance curves for the same solutions indicates clearly a close relationship between conductance and specific refraction.

\section{Summary.}

(I) The conductance of methyl and ethyl alcoholic solutions of cadmium iodide at $25^{\circ}$ has been measured and found to be in certain respects abnormal.

(2) These abnormalities have been shown to be similar to those previously observed in certain nonaqueous solutions of silver nitrate and a similar explanation has been assumed.

(3) The densities and refractive indices of these solutions have been measured at $20.5^{\circ}$ and it has been shown that each is a rectilinear function of the concentration.

(4) The specific refraction calculated by the Lorentz-Lorenz formula has revealed certain abnormalities.

(5) A close connection has been pointed out between conductance and specific refraction.

BRYN MAWR, PA. 